Check for updates

Cite this: Chem. Commun., 2020, 56,7143

Received 5th May 2020,

Accepted 21st May 2020

DOI: $10.1039 / \mathrm{d} 0 \mathrm{cc} 03217 \mathrm{~d}$

rsc.li/chemcomm

\section{Unveiling the mechanism of hydrotropy: evidence for water-mediated aggregation of hydrotropes around the solute $\dagger$ t}

\author{
Dinis O. Abranches, (D) a Jordana Benfica, (D) a Bruna P. Soares, (D) a \\ Alejandro Leal-Duaso, id b Tânia E. Sintra, (iD a Elísabet Pires, (iD b Simão P. Pinho, (iD c \\ Seishi Shimizu (D) ${ }^{d}$ and João A. P. Coutinho (DD*a
}

\begin{abstract}
A recent proposal attributes the origin of hydrotropy to the watermediated aggregation of hydrotrope molecules around the solute. Experimental evidence for this phenomenon is reported for the first time in this work, using ${ }^{1} \mathrm{H}-\mathrm{NMR}$. A new computational technique to quantify apolarity is introduced and is used to show that apolarity of both solute and hydrotrope is the driving force of hydrotropy.
\end{abstract}

Hydrotropes, in their ability to increase the solubility of hydrophobic substances in water, can expand the applicability of the greenest and most abundant of all solvents. Broadening the repertoire of safer solvents is in line with the principles of green chemistry $^{1}$ and is essential for a sustainable future. ${ }^{2-4}$ Besides, the addition of water suffices to force the precipitation of a solute dissolved in a hydrotropic solution, facilitating its purification. ${ }^{5,6}$

The mechanism of hydrotropy is still not clearly understood, despite a century of research ${ }^{7}$ and a large debate in the literature. ${ }^{8-17}$ Traditional speculations regarding the mechanism of hydrotropy revolved around (i) bulk-phase self-aggregation (or pre-clustering) of hydrotropes analogous to micellar solubilization, ${ }^{8,9,17}$ (ii) "water structure" disruption by the hydrotrope that would behave like chaotropic agents weakening the hydrophobic effect ${ }^{10,11}$ and (iii) specific stoichiometric association between solute and hydrotrope. ${ }^{12,13}$ However, none of these hypotheses are supported by statistical thermodynamics

\footnotetext{
${ }^{a}$ CICECO - Aveiro Institute of Materials, Department of Chemistry, University of Aveiro, 3810-193 Aveiro, Portugal. E-mail: jcoutinho@ua.pt

${ }^{b}$ Instituto de Sintesis Quimica y Catálisis Homogénea (ISQCH-CSIC) Facultad de Ciencias, C. S. I. C. - Universidad de Zaragoza, E-50009 Zaragoza, Spain ${ }^{c}$ Centro de Investigação de Montanha (CIMO), Instituto Politécnico de Bragança, Campus de Santa Apolónia, 5300-253 Bragança, Portugal

${ }^{d}$ York Structural Biology Laboratory, Department of Chemistry, University of York, Heslington, York YO10 5DD, UK

$\dagger$ Dedicated to the memory of Prof. José Ignacio García Laureiro.

‡ Electronic supplementary information (ESI) available: Experimental and computational details and supporting figures, including NMR and apolar factor results. See DOI: 10.1039/d0cc03217d
}

descriptions of hydrotropy ${ }^{14,15}$ which suggest that hydrotrope accumulation around the solute is driven by a strong watermediated (or hydrophobic) interaction between hydrotrope and solute. Because the apolar (or hydrophobic) moiety of a molecule interacts with water much weaker than a water-water hydrogen bond, it is driven out to associate with another hydrophobic moiety, resulting in strong agglomeration of hydrotrope around the solute.

Despite stemming from the principles of statistical thermodynamics and its consequent superiority, in terms of theoretical grounds, to the previous hypothesis, no direct experimental evidence has been to this day reported for the water-mediated accumulation mechanism proposed. In this work it will be shown that (1) the hydrophobic interaction between a hydrotrope and a solute is the driving force for the accumulation and that (2) such interaction can be quantified via a measure for apolarity derived using COSMO-RS. Both results are crucial to understand the mechanism of hydrotropy and support the hypothesis of strong water-mediated solute-hydrotrope apolar interactions.

Proton nuclear magnetic resonance $\left({ }^{1} \mathrm{H}-\mathrm{NMR}\right)$ is herein employed to provide experimental evidence for hydrotropesolute aggregation. The strategy is based on the well-known principle that the chemical shifts of the protons of a molecule dissolved in water may change due to the presence of another substance. ${ }^{6,18}$ More precisely, a chemical shift that diminishes in the presence of another substance infers a higher shielding of that proton or a less probable contact between it and water. Thus, the chemical shifts of the protons associated to apolar moieties (namely methyl groups) of a hydrotrope dissolved in water can be measured in the presence and absence of a solute. If the solute induces the aggregation of hydrotropes around itself through water-mediated apolar interactions, as is predicted by statistical thermodynamics, the chemical shift of the protons associated to the apolar moieties of the hydrotrope should decrease.

Gallic acid and syringic acid were chosen as solutes and monoalkylglycerol ethers, listed in Table S1 (ESI $\ddagger$ ), as hydrotropes. 
Note that the apolarity of monoalkylglycerol ethers can be made to vary smoothly through the progressive increase in the length of their alkyl chain. Likewise, gallic acid and syringic acid are structurally similar but present different hydrophobicities. Furthermore, Kunz and co-authors pointed out the necessity to study this class of hydrotropes. ${ }^{16}$ The solubilities of both acids in monoalkylglycerol ether aqueous solutions have been reported elsewhere. ${ }^{5}$

For each hydrotrope-solute pair, ${ }^{1} \mathrm{H}-\mathrm{NMR}$ spectra were acquired thrice. In each case, the concentration of hydrotrope was maintained $\left(0.4 \mathrm{~mol} \mathrm{\textrm {kg } ^ { - 1 }}\right)$ but solute concentration was changed, from zero solute, which serves as reference for the chemical shifts of the hydrotrope, to concentrations below and above solute solubility in pure water (Table S2, ESI $\$$ ). The NMR peaks considered, clearly marked in Fig. S1 of ESI, $\$$ were those of the protons of the alkyl side chain of the monoalkylglycerol ether along with the sole proton of the second carbon of the glycerol head. The protons of the first and third carbon of the glycerol head were not analysed due to the difficulty of distinguishing their peaks in the NMR spectra.

The results obtained using gallic acid as a solute (Fig. 1a as an illustrative example and Fig. S2, ESI $\$$ ) show that the chemical shift of the protons diminishes as the concentration of solute increases. This means that the apolar moieties of the hydrotropes are statistically less prone to interact with water, providing evidence for the idea of association between these moieties and the apolar moieties of the solute. Moreover, the decrease in chemical shift seen in Fig. 1a and Fig. S2 (ESI $\ddagger$ ) is proportional to the concentration of solute. This is expected since if more solute is present in the system, more hydrotrope is needed to interact with it. Because syringic acid is much less soluble in water, its concentration was one order of magnitude lower than that of gallic acid in the NMR experiments, leading to smaller changes in the chemical shifts of the hydrotrope. In some cases, the changes produced are comparable to the experimental uncertainty of the technique (ca. $0.002 \mathrm{ppm}$ ). Nevertheless, the conclusions taken from the NMR results of gallic acid hold true for syringic acid as well, as depicted in Fig. 1b and Fig. S3 (ESI $\$$ ).
The NMR results question the idea that pre-clustering of the hydrotrope is fundamental in hydrotropy. Hydrotropes unquestionably do possess a degree of aggregation (clustering) with themselves. However, the hydrotrope aggregation (whether it is present depends on the system) clearly changes with the addition of the solute, providing clear evidence that the solute is not merely entering a "micelle"-like bulk-phase pre-clustering of the hydrotrope. If this were the case, there should be no change in the chemical shifts of the hydrotrope protons, since alternating from a previously hydrotrope-hydrotrope contact to a hydrotropesolute contact would not make the hydrotrope less prone to interact with water, hence would not lead to a decrease in the chemical shifts.

Insight is given by Fig. 1 and Fig. S2, S3 (ESI $\ddagger$ ) not only into its existence but also into the geometry of aggregation. In fact, the peak assigned to the protons of the second carbon of the hydrotrope always shifts less than the remaining peaks. This means that the second carbon is less prone to aggregation, which is explained by its higher degree of polarity, brought about by the presence of hydroxyl groups in its vicinity. Moreover, for all systems, the peak assigned to the protons in the last methyl group of the side alkyl chain is consistently the second less-shifting peak. That is, the second less-shifting peak for [2.0.0] is that of carbon 5, for [3.0.0] is that of carbon 6 , for [4.0.0] is that of carbon 7 and for [5.0.0] is that of carbon 8 (see insets of Fig. S1 (ESI $\neq$ ) for clarification). This can be interpreted in terms of interaction geometry; a parallel contact between hydrotrope and solute covers more apolar area, thus being more energetically favourable to water, than a hydrotrope tail-solute contact.

It has been argued that, for certain types of apolar yet slightly hydrophilic solutes, hydrotropy could occur due to hydrotrope-solute interactions through their polar functional groups. ${ }^{17}$ This is clearly not the case for the solutes and hydrotropes studied in this work, since NMR spectroscopy revealed that the aggregation of hydrotrope around the solute happens through apolar contacts, even giving information about the geometry of these contacts.
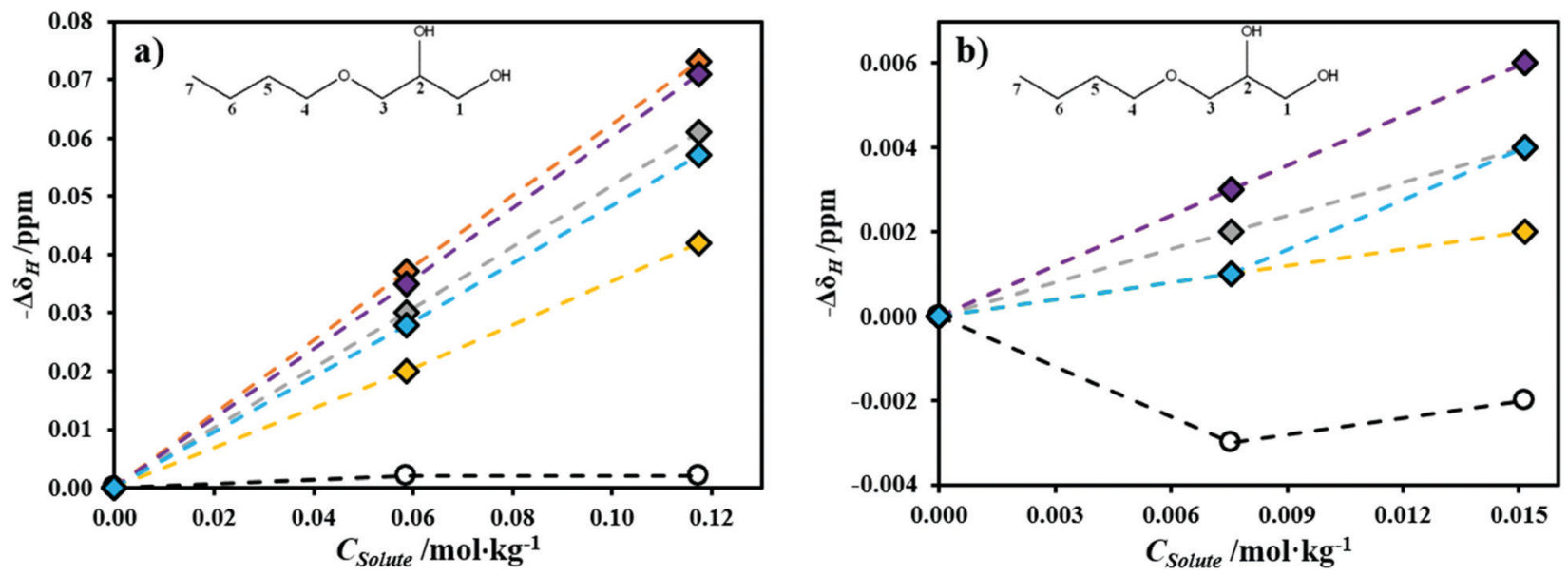

Fig. 1 Change in chemical shift of the protons $\left(-\Delta \delta_{\mathrm{H}}\right)$ associated to water and several methyl groups of [4.0.0] (structure as inset) dissolved in water $\left(0.4 \mathrm{~mol} \mathrm{~kg}{ }^{-1}\right)$ as a function of (a) gallic acid or (b) syringic acid concentration. Legend: - $\bigcirc-$ water; $\diamond-2$ nd carbon; $\diamond$ th carbon; $-\diamond$ - 5th carbon; $\diamond$ - 6th carbon; $\diamond$ - 7th carbon. 
Having provided direct, experimental evidence for the hydrotropesolute aggregation, we now address its driving force. As a quantitative measure for the apolarity of a molecule, the unnormalized $\sigma$-profile framework of COSMO-RS has been adopted. ${ }^{19}$ This is a histogram representing the amount of molecular surface with a given polarization charge-density, $\sigma$. The unnormalized $\sigma$-profile framework should prove advantageous for the study of hydrotropy since it can quantify the apolarity of both hydrotrope and solute. Moreover, the geometry and polarity of molecules optimized within the COSMO solvation model should more closely resemble that which is found in a real aqueous solution than molecules optimized in the gas phase. Details for the optimization, using TURBOMOLE V7.1, of the solute and hydrotrope molecules herein studied are given in ESI. $\$$

To study the relationship between apolarity and hydrotropy using the $\sigma$-profile framework, an apolar factor was defined:

$$
\int_{-0.0082}^{0.0082} p(\sigma) \cdot(0.0082-|\sigma|) \cdot \mathrm{d} \sigma
$$

This factor is a measure of the area under the curve of the apolar region, with the apolar/polar limit being defined as

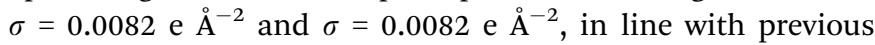
studies. ${ }^{19,20}$ The amount of apolar surface area, $p(\sigma)$, is weighted by the actual polarity of the surface, with regions near the apolar/polar limit being progressively penalized by the term $(0.0082-|\sigma|)$. The Setschenow constant (the ratio between solubility enhancement and hydrotrope concentration in the dilute region) of several hydrotropic systems previously reported in the literature was correlated against this apolar factor. Table S 3 of the ESI, $\neq$ contains the apolar factors of these hydrotropes while the correlations obtained are reported in Fig. 2 for glycerol ether systems and Fig. S4 (ESI $\neq$ ) for systems taken from the literature where the Setschenow constants were reported for different hydrotropes but the same solute. The results obtained show that, for the same family of hydrotropes, the solubility enhancement of the solute positively correlates with the apolar factor of the hydrotrope. This supports the view of water-mediated aggregation of hydrotrope around the solute and supports the idea of hydrotrope apolarity as the driving force of hydrotropy.

Shimizu and Matubayasi ${ }^{21}$ derived a hydrotropy model based on cooperative water-mediated hydrotrope-solute aggregation using statistical thermodynamics. When regressed against experimental solubility curves, this model returns the average number of hydrotrope molecules in the vicinity of the solute (parameter $m$ ). Since this model was previously applied to the systems herein studied, ${ }^{5}$ Fig. 3 depicts this parameter plotted against the apolar factor of the corresponding hydrotrope. The resulting plot shows that $m$ reaches a maximum for both gallic acid and syringic acid. Surprisingly, this maximum is located at the apolar factor of the solute. As described above, solute-hydrotrope interactions are established between their apolar moieties, resulting in strong and favourable interactions only due to the presence of water. However, there is no distinction between apolar moieties of solute and hydrotrope. Consequently, a hydrotrope that is more apolar than the solute will tend to agglomerate with itself more promptly than with the solute. Put differently, in terms of apolar contacts, it is as if there are three different forms of hydrotrope present in the system: free hydrotrope, hydrotrope associated with solute and hydrotrope associated with itself. This, again, disputes the pre-clustering hypothesis, since more self-aggregation of the hydrotrope leads to less aggregation around the solute.

Note that links between hydrophobicity of the hydrotrope and the extent of hydrotropy have been proposed before. ${ }^{5,8,16,17}$ However, this is the first time that these parameters (hydrotropy extent and apolarity of the hydrotrope) are quantified and shown to correlate remarkably well with each other. Furthermore, it is shown that the apolarity of the hydrotrope is not the only factor influencing hydrotropy. In fact, the driving force for aggregation is the balance between apolarity of both solute and hydrotrope, as demonstrated by Fig. 3 .

Fig. 3 shows that hydrotropes that are more apolar than the solute will tend to aggregate less around it. This does not translate, however, into a maximum on the solubility enhancement (Fig. 2) for the case of glycerol ethers. This is rationalized by taking into account that even though the most apolar hydrotropes
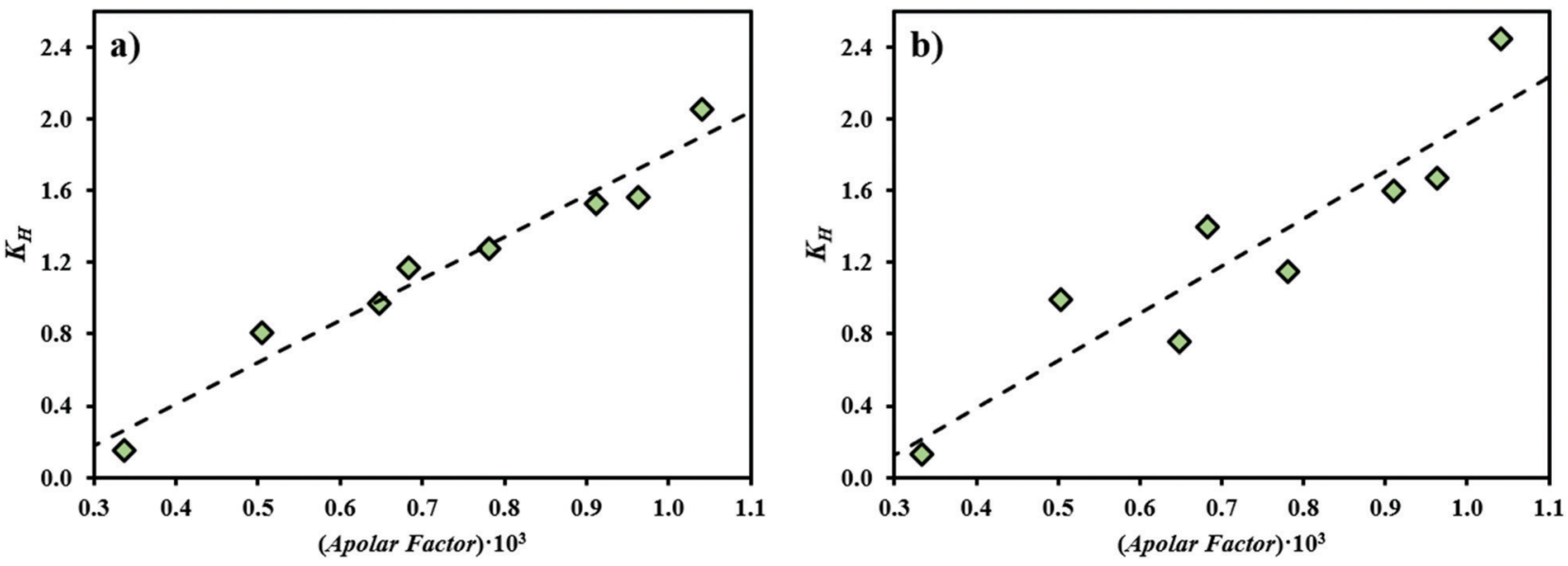

Fig. 2 Setschenow constants ${ }^{16}$ for (a) gallic acid or (b) syringic acid in glycerol ether-based hydrotropic solutions as a function of the apolar factor of the hydrotrope. The dashed line is the straight line fitted to the data using the least squares method. 

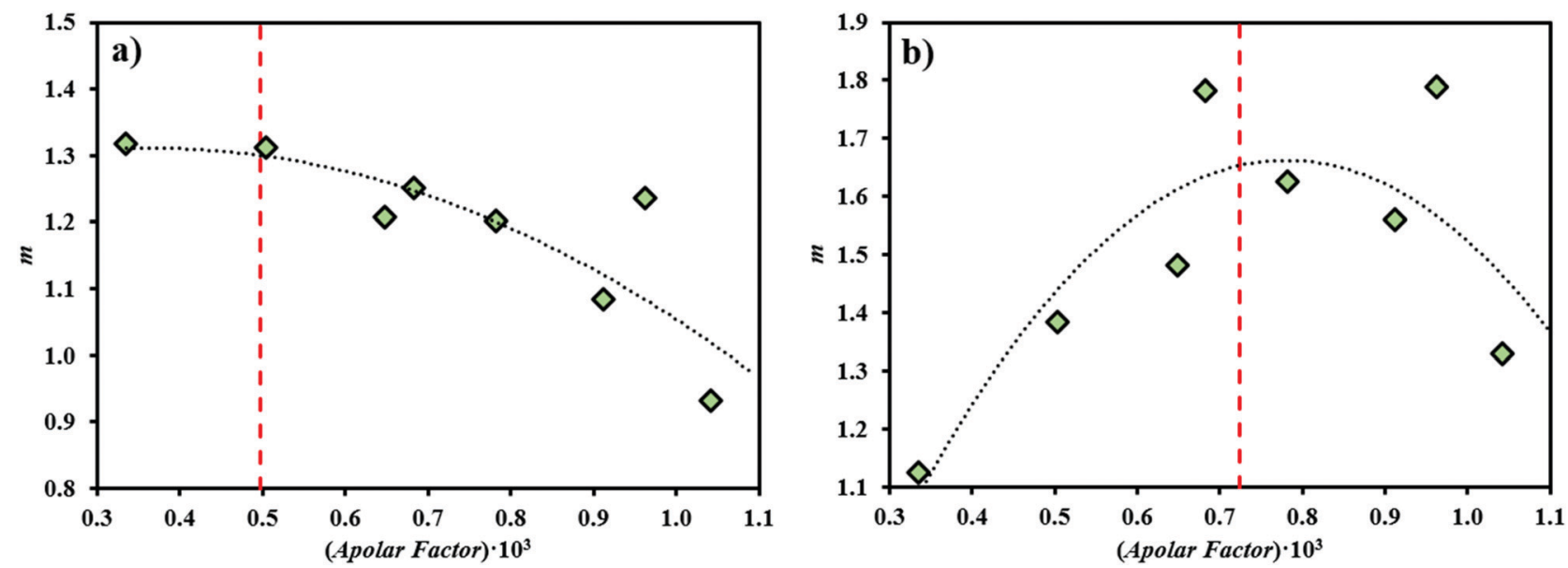

Fig. 3 Dependency of the number of hydrotrope molecules in the vicinity of the solute $(\mathrm{m})$ on the apolar factor of the hydrotrope, estimated using the Shimizu and Matubayasi ${ }^{19}$ model for (a) gallic acid and (b) syringic acid in aqueous solutions of glycerol ethers. ${ }^{16}$ The black dashed line is a visual guide whilst the red dashed line represents the apolar factor of the solute.

may statistically possess less molecules around the solute, they are able to cover more of its apolar area due to their larger size and chain linearity, and, thus, increase its solubility.

In conclusion, experimental evidence based on ${ }^{1} \mathrm{H}-\mathrm{NMR}$ chemical shifts is here reported for the first time showing that hydrotrope molecules aggregate around the solute, which supports the cooperativity theory of hydrotropy. Moreover, it was shown that apolarity of both hydrotrope and solute is the driving force of hydrotropy, with strong solute-hydrotrope interactions arising in the presence of water. These interactions are statistical and are established between apolar moieties of both solute and hydrotrope, instead of interactions between their polar functional groups. These water-mediated interactions are, however, not exclusive to solute-hydrotrope pairs and the number of hydrotropes aggregated around the solute is maximum when the apolarity of hydrotrope and solute is the same. The results reported in this work are, thus, of the utmost importance in the understanding of the water-mediated hydrotrope-solute interactions hypothesis and provide the necessary background to design new hydrotrope molecules for specific applications. A schematic illustration of the mechanism of hydrotropy, in light of these findings, is provided in Fig. S5 (ESI $\ddagger$ ).

This work was developed within the scope of the projects CICECO-Aveiro Institute of Materials, UIDB/50011/2020 \& UIDP/50011/2020, and CIMO-Mountain Research Center, UIDB/00690/2020, both financed by national funds through the FCT/MEC and when appropriate co-financed by FEDER under the PT2020 Partnership Agreement. The NMR spectrometers are part of the National NMR Network (PTNMR) and are partially supported by Infrastructure Project No. 022161 (co-financed by FEDER through COMPETE 2020, POCI and PORL and FCT through PIDDAC). Financial support from Ministerio de Ciencia, Innovación (project RTI2018-093431-B-I00) and the Gobierno de Aragón (Group E37_17R) co-funded by FEDER 2014-2020 "Construyendo Europa desde Aragón” is acknowledged. B. P. S. acknowledges FCT for her PhD grant SFRH/BD/138439/2018.

\section{Conflicts of interest}

There are no conflicts to declare.

\section{Notes and references}

1 P. Anastas and N. Eghbali, Chem. Soc. Rev., 2010, 39, 301-312.

2 P. T. Anastas and J. B. Zimmerman, Green Chem., 2019, 21, $6545-6566$.

3 P. G. Jessop, Green Chem., 2011, 13, 1391.

4 M. Cvjetko Bubalo, S. Vidović, I. Radojčić Redovniković and S. Jokić, J. Chem. Technol. Biotechnol., 2015, 90, 1631-1639.

5 B. P. Soares, D. O. Abranches, T. E. Sintra, A. Leal-Duaso, J. I. García, E. Pires, S. Shimizu, S. P. Pinho and J. A. P. Coutinho, ACS Sustainable Chem. Eng., 2020, 8, 5742-5749.

6 A. F. M. Cláudio, M. C. Neves, K. Shimizu, J. N. Canongia Lopes, M. G. Freire and J. A. P. Coutinho, Green Chem., 2015, 17, 3948-3963.

7 C. Neuberg, Biochem. Z., 1916, 76, 107-108.

8 P. Bauduin, A. Renoncourt, A. Kopf, D. Touraud and W. Kunz, Langmuir, 2005, 21, 6769-6775.

9 J. Y. Kim, S. Kim, M. Papp, K. Park and R. Pinal, J. Pharm. Sci., 2010, 99, 3953-3965.

10 R. E. Coffman and D. O. Kildsig, J. Pharm. Sci., 1996, 85, 951-954.

11 H. S. Frank and F. Franks, J. Chem. Phys., 1968, 48, 4746-4757.

12 R. Sanghvi, D. Evans and S. H. Yalkowsky, Int. J. Pharm., 2007, 336, 35-41.

13 Y. Cui, Int. J. Pharm., 2010, 397, 36-43.

14 J. J. Booth, M. Omar, S. Abbott and S. Shimizu, Phys. Chem. Chem. Phys., 2015, 17, 8028-8037.

15 J. J. Booth, S. Abbott and S. Shimizu, J. Phys. Chem. B, 2012, 116, 14915-14921.

16 W. Kunz, K. Holmberg and T. Zemb, Curr. Opin. Colloid Interface Sci., 2016, 22, 99-107.

17 T. Buchecker, S. Krickl, R. Winkler, I. Grillo, P. Bauduin, D. Touraud, A. Pfitzner and W. Kunz, Phys. Chem. Chem. Phys., 2017, 19, 1806-1816.

18 A. Stark, M. Sellin, B. Ondruschka and K. Massonne, Sci. China: Chem., 2012, 55, 1663-1670.

19 A. Klamt, COSMO-RS: From Quantum Chemistry to Fluid Phase Thermodynamics and Drug Design, Elsevier Science, 1st edn, 2005.

20 J. Palomar, J. S. Torrecilla, J. Lemus, V. R. Ferro and F. Rodríguez, Phys. Chem. Chem. Phys., 2010, 12, 1991.

21 S. Shimizu and N. Matubayasi, Phys. Chem. Chem. Phys., 2016, 18, 25621-25628. 